International Journal of Engineering \& Technology, $7(2.8)(2018) 468-471$
International Journal of Engineering \& Technology
SPC
Website: www.sciencepubco.com/index.php/IJET
Research Paper

\title{
Comparative study of image enhancement techniques using histogram equalization on degraded images
}

\author{
Shalika Arora*, Megha Agarwal, Veepin kumar, Divya Gupta \\ Department of Computer Science and Engineering, SRM Institute of Science and Technology, NCR Campus, Modinagar (India) \\ *Corresponding Author Email: shalika.chhabra20@gmail.com
}

\begin{abstract}
Image Enhancement technique plays a vital role in digital image processing for making an image to be useful for various applications. This technique is used to improve the quality of degraded images. Usually, the degradation is not evenly spread throughout the image, but most of the time it varies from region to region. Our aim is to first identify the region where enhancement is required and improve that region without disturbing its neighbourhood which does not require any improvement.
\end{abstract}

Keywords: Image Enhancement, Image Histogram, Histogram Equalization (HE), Contrast Limited Adaptive Histogram Equalization (CLAHE).

\section{Introduction}

Nowadays, everything is becoming digital and to maintain the quality of digital images become the main concern of researchers doing research in image processing which in turn leads to the invention of image enhancement technique. Image Enhancement techniques isused to enhance the resolution and the clarity of image[1].The output of digital image processing sensor is a "raw" digital image that consists of large number of values and each value represents the brightness of a pixel in the image. The working of image processing is illustrate with the help of below given Fig 1.

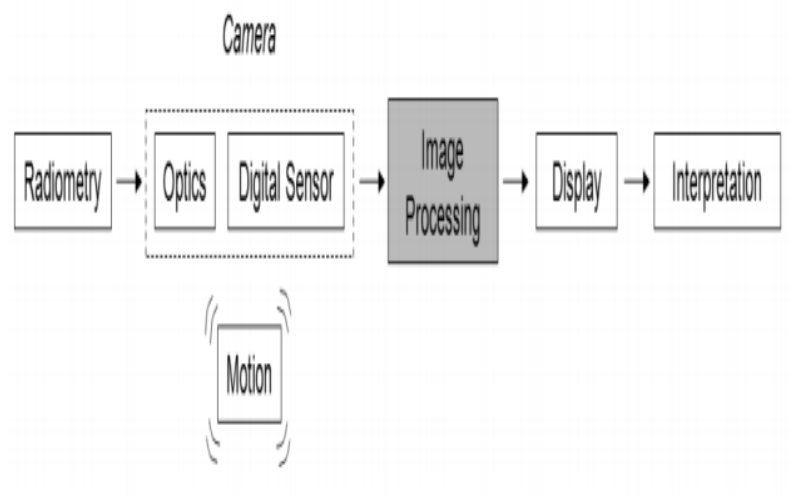

Fig.1: Image Processing

\subsection{Thresholding}

Thresholding is one of the important preprocessing techniques used for enhancing the quality of degraded images.

Threshold value is used to separate the required region of the image from the background region. Background region can be extracted from the image on the basis of the distribution of gray levels in image. Thresholding is used to divide an image by setting all pixels whose intensity values are above a threshold to a foreground value and all the remaining pixels to a background
Value [3]. In Thresholding techniques black pixel corresponds to the background and white pixel corresponds to the foreground details(or vice-versa) in the image.

When function depends only on gray-level values then the threshold is called local threshold and if it depends on the local property of image then the threshold is called dynamic threshold [6].

In this paper, we try to improve the quality of degrade images using histogram equalization algorithms. Here we discuss two existing histogram equalization technique (HE and CLAHE) and compare with proposed algorithm. And at last, compare all of these algorithms with each other and evaluate the best one.

\section{Histogram Equalization}

A histogram of an image is the bar graph that helps visualizing its intensity distribution. The initial step of drawing a histogram is to collect the data and sort it into the categories. The independent variable is represented at the horizontal axis of histogram and the vertical axis represents the dependent variable.

The operator which is closely connected to the histogram is histogram Equalization. By equalizing the image histogram, the quality of image can be enhanced. Histogram equalization is an image processing technique which works on enhancing visual appearance of an image by uniformly distributing the intensity levels. It normally enhances the contrast of the image globally, especially when the usable data of the image is represented by close contrast values. By using this equalizing concept, the intensity levels of the image can be uniformly distributed in the histogram. In this way, the local contrast of the image is enhanced without affecting the global contrast of the image[7]. Histogram equalization accomplishes this by effectively spreading out the most frequent intensity values[5]. If the histogram of any image has many peaks and valleys, then the image obtained after equalization can stilll have peaks and valleys, but they will be uniformly distributed. In histogram equalization, each pixel is assigned a new intensity value based on the transformation function applied on its previous intensity level [6]. 


\subsection{Adaptive Histogram Equalization}

There are several local image enhancement techniques. In this paper, we basically discuss two image enhancement techniques i.e. adaptive histogram equalization(AHE) and Contrast limited adaptive histogram equalization(CLAHE) technique. Adaptive histogram equalization is outstanding contrast enhancement technique. In AHE, it calculates different histogram, each histogram belongs to a different region of image and after that it redistributes the contrast of the image. There are two limitations of adaptive histogram equalization i.e., slow speed on computer and over-enhancement of noise.

The substitute method of AHE is CLAHE. It overcomes the problem of over-enhancement of noise which is present in AHE.

Traditionally, Histogram Equalization technique uses the same transformation method (obtained from image histogram)to transform all the pixels of the image. This technique gives best results when the pixel values are uniformly distributed in the image. On the other hand, the contrast of the image is not sufficiently enhanced if the image is having lighter or darker regions.

Adaptive histogram equalization (AHE) uses the transformation function (obtained from neighbourhood region) to transform all the pixels of the image. The transformation function is directly proportional to the cumulative distribution function of pixel values in the neighbourhood region.

\section{Problem Formulation}

In the existing system, while applying histogram equalization along with global enhancement of the image, it also amplifies the impact of noise pixels in the background. As a result, the noise that was not so prominent in the input image can now be noticed significantly after the enhancement is done, hence failing the purpose of the whole process. It also often tends to wash out background details in the image after the equalization is done. This issue can be resolved by AHE and CLAHE technique since they do not increase the contrast globally but perform it locally. Although they greatly limit the amplification of noise but most of the time fail to raise the contrast as significantly as their global counterpart.

\subsection{Proposed Algorithm}

In existing algorithms discussed above, the former tends to over amplify input and the latter under amplifies in order to overcome the side effects of over amplification. The present work tries to find a middle ground between the two algorithms toper form the enhancement or amplification to a level such that it is neither too much nor too less. The algorithm as depicted using flowchart shown in Fig 2 considers that the enhancement is not necessarily required on the complete image but on some regions in the image and only those regions should be enhanced leaving the other regions unaffected. The steps to be followed as:-

1. Initially, a loaded image is converted into a gray scale image.

2. On the image obtained in the above step, apply the threshold operator by using an exposure threshold. This results into an image having over-exposure and under-exposure components.

3. Perform clipping on the thresholded image.

4. Adaptive histogram equalization (AHE) is performed on the sub divisions to enhance the contrast of only those regions in the image that require it.

5. Output of the above step is the final enhanced image

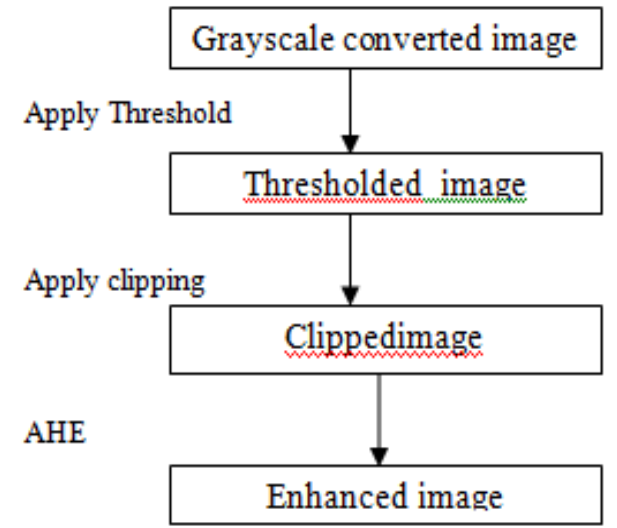

Fig. 2: Flowchart of proposed algorithm

\section{Results}

This paper describes the results and comparison of the results with other existing systems i.e. Histogram Equalization (HE) approach and Contrast Limited Adaptive Histogram Equalization (CLAHE) approach.

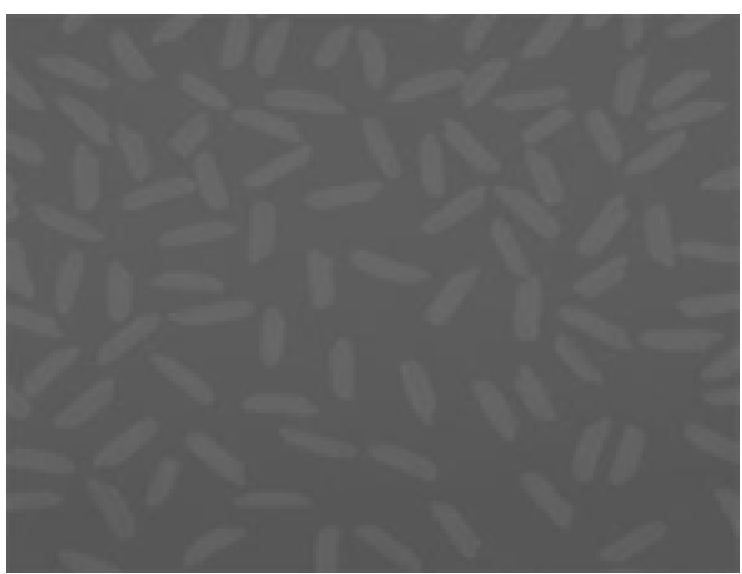

Fig. 3: Input Image 1

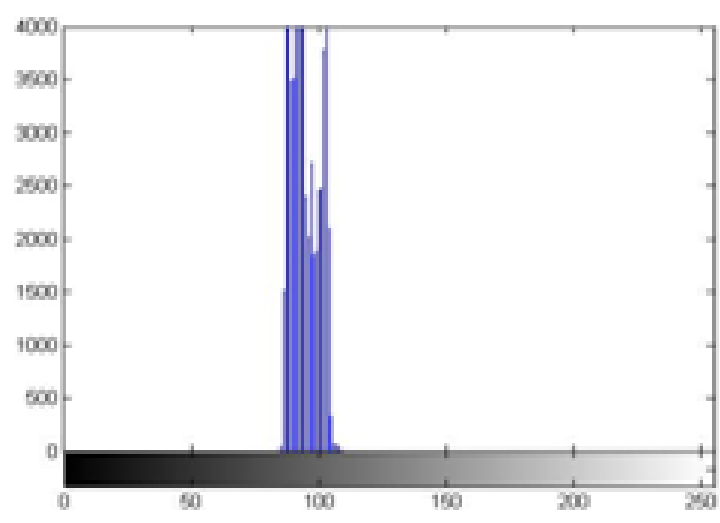

Fig. 4: Histogram of input image 1 


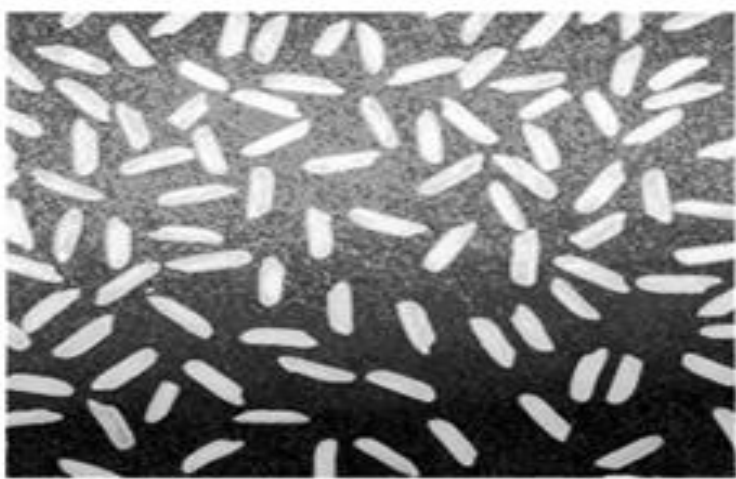

Fig.5: HE image1

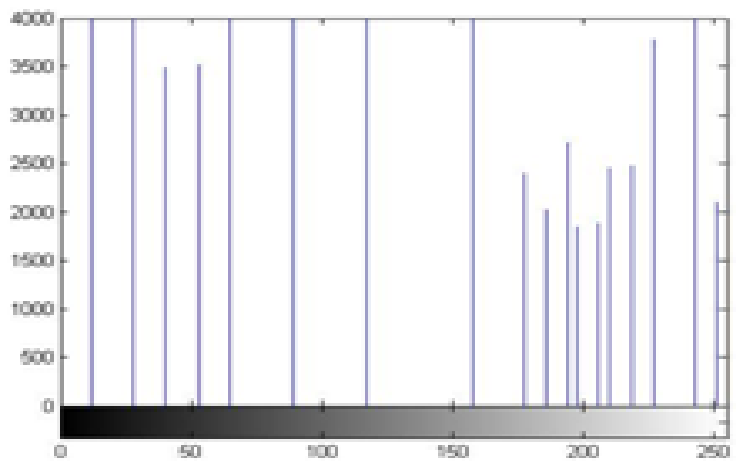

Fig 6: Histogram of HE image 1

Histogram equalization increases the contrast significantly but the noise present in the input image is also amplified in the process. This noise amplification effect can be seen in Fig 4. The HE image is the enhanced image after performing Histogram Equalization technique on input image. It can be seen that the black background is inconsistent due to introduction of noise in it.

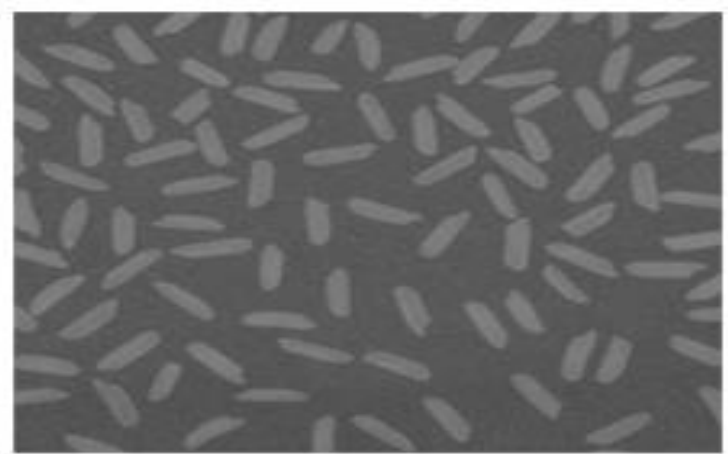

Fig. 7: CLAHE image 1

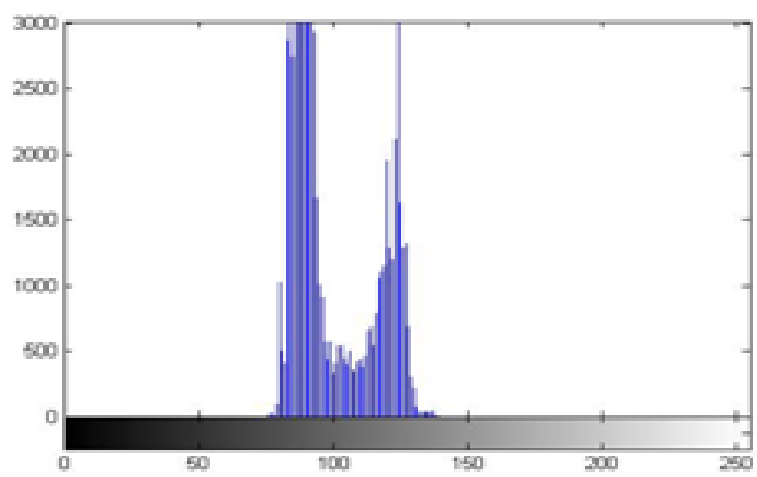

Fig. 8: Histogram of CLAHE image

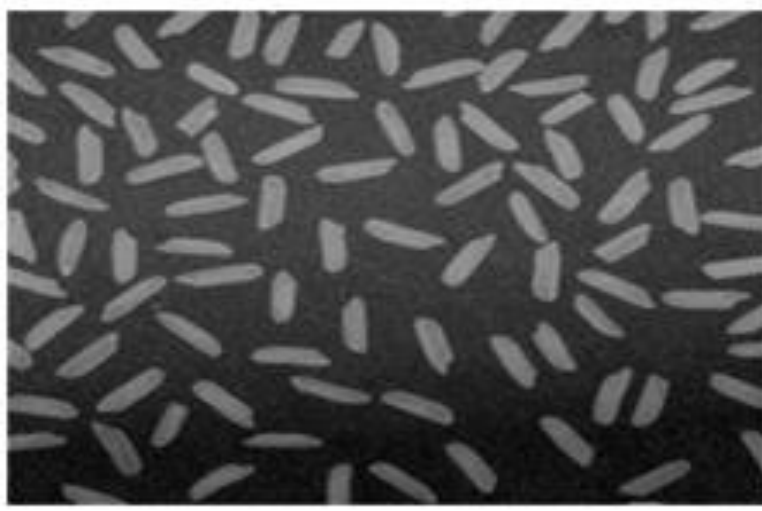

Fig. 9: Proposed 1

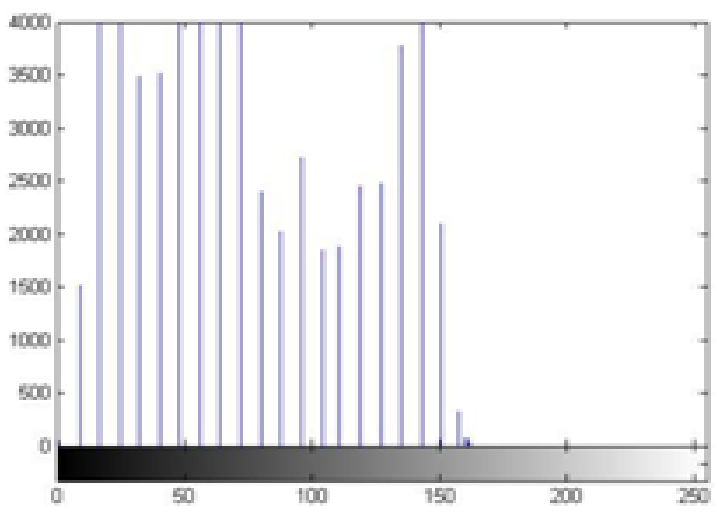

Fig. 10: Histogram of Proposed 1

Fig. 7 shows that when Contrast Limited Adaptive Histogram Equalization (CLAHE) approach is applied on the digital image, the resultant avoids the noise amplification but this is achieved at the cost of limiting the contrast amplification. Hence the output image lacks significant gains in contrast. Fig. 8 shows the histogram of the CLAHE image. The output of the proposed technique is shown in Fig. 9.It is clear from the output image that contrast amplification is higher than the CLAHE and the background noise is also kept under control compared to the HE image.

\section{Conclusion}

Image enhancement is a crucial step in digital image processing. Few image enhancement techniques, such as HE and CLAHE, were studied and a new and image enhancement algorithm is proposed, which will enhance images and also restricts noise amplification. The results obtained from the proposed technique are compared with existing enhancement solutions.

The results obtained above are satisfactory and shows that the proposed technique can improve the contrast of the images efficiently, keeping the background noise in check at the same time. The other merit of the proposed technique is that it requires no parameter settings.

\section{References}

[1] Rashmi Choudhary and Sushopti Gawade, "Survey on Image Contrast Enhancement Techniques", International Journal of Innovative Studies in Sciences and Engineering Technology (IJISSET), Volume 2, Issue 3, March 2016,pp.21-25.

[2] Senthilkumaran N and Vaithegi S, "Image Segmentation by using Thresholding techniques for medical images", Computer Science \& Engineering: An International Journal (CSEIJ), Vol.6, No.1, February 2016,pp.1-13. 
[3] Sangeeta Rani, Ashwini Kumar and Kuldeep Singh,"Illumination based Sub Image Histogram Equalization: A Novel Method of Image Contrast Enhancement, International Journal of Computer Applications (0975 - 8887) Volume 119, No.20, June 2015,pp.14-19.

[4] Rima Deb, Dipali Sengupta, Rupasree Das, Subhankar Mahajan and Sanjoy Mitra"Development of an Image Enhancement Method and a GUI", , International Journal of Signal Processing, Image Processing and Pattern Recognition Vol.8, No.6, 2015,pp.227-234.

[5] S.S. Bedi and Rati Khandelwal, "Various Image Enhancement Techniques - A Critical Review", International Journal of Advanced Research in Computer and Communication Engineering Vol. 2, Issue 3, March 2013,pp.1605-1609.

[6] Ms. Seema Rajput and Prof. S. R. Suralkar, "Comparative Study of Image Enhancement Techniques", International Journal of Computer Science and Mobile Computing Vol. 2, Issue. 1, January 2013,pp.11-21.

[7] Ms. K.T.Chalekar and Prof T. Yengantiwar, "Review paper on image contrast enhancement techniques", International Journal of Advanced Research in Computer Engineering \& Technology (IJARCET) Volume 3 Issue 3, March 2014,pp.909-911.

[8] Rafael C. Gonzalez (University of Tennessee), Richard E. Woods (MedData Interactive) and Steven L. Eddins (The MathWorks, Inc.), in 'Digital Image Processing Using MATLAB' Second Edition,2009 by Gatesmark, LLC.

[9] Arunkumar, T.S. Sivakumaran, K. Ramash Kumar, "Improved Performance of Linear Quadratic Regulator plus Fuzzy Logic Controller for Positive Output Super Lift Luo-Converter," Journal of Electrical Engineering, Vol. 16, Edition:3, 2016, pp. 397-408.

[10] Harikishore Kakarla, Madhavi Latha M and Habibulla Khan, "Transition Optimization in Fault Free Memory Application Using Bus-Align Mode", European Journal of Scientific Research, Vol.112, No.2, pp.237-245, ISSN: 1450216x135/1450-202x, October 2013.

[11] T. Padmapriya, V.Saminadan, "Performance Improvement in long term Evolution-advanced network using multiple imput multiple output technique", Journal of Advanced Research in Dynamical and Control Systems, Vol. 9, Sp-6, pp: 990-1010, 2017.

[12] S.V.Manikanthan and D.Sugandhi " Interference Alignment Techniques For Mimo Multicell Based On Relay Interference Broadcast Channel " International Journal of Emerging Technology in Computer Science \& Electronics (IJETCSE) ISSN: 0976-1353 Volume- 7 ,Issue 1 -MARCH 2014. 